\title{
An Efficient Graph-Based Algorithm for Fingerprint Representation and Matching
}

\author{
Xiaoguang Chen ${ }^{1}$, Lin Wang ${ }^{2}$ and Mingyan $\mathrm{Li}^{3}$
}

\begin{abstract}
This paper presents a novel graph-based algorithm for robust fingerprint recognition, which efficiently improves the approach "K-plet and CBFS" proposed by Chikkerur et al at ICB'06. To cope with the crucial problems of nonlinear distortion and partial overlap, a new feature called supplementary associative point $(S A P)$ is extracted to provide additional discriminative information for representation and matching. The local spatial relationship of the improved K-plets, which are constructed by minutiae and SAPs, is encoded as a directed graph to represent the fingerprint. Besides, an improved CBFS-based matching algorithm is also proposed. It utilizes the consistent matching property of minutia-SAP pairs, and possesses advantages such as fast pruning of imposter local matches and deep propagation of local matches in graph. Experiments on FVC database show that the proposed algorithm is strongly robust to nonlinear distortion and partial overlap, and outperforms the original algorithm with an average improvement of $24.17 \%$ on EER.
\end{abstract}

Keywords: Fingerprint representation - Fingerprint minutiae matching $\bullet$ Supplementary associative point $\bullet$ K-plet $\bullet$ CBFS

\footnotetext{
${ }^{1}$ X. Chen $(\bowtie)$

Institute of Image Processing and Pattern Recognition, College of Sciences, North China

University of Technology, Beijing 100144, China

e-mail: chenxg1018@sina.com

${ }^{2}$ L. Wang $(\square)$

Department of Mathematics, College of Sciences, North China University of Technology,

Beijing 100144, China

${ }^{3}$ M. Li ( $\left.\square\right)$

College of Sciences, Beijing Forestry University, Beijing 100083, China
} 


\section{Introduction}

Nowadays, fingerprint-based biometrics has been widely used in personal identification applications [1]. In automatic fingerprint recognition systems (AFRS), the matching algorithm is the most important stage, and it compares two fingerprints and computes a similarity score indicating the probability of being from the same fingertip. During last several decades, various algorithms have been proposed for matching two fingerprints. In these algorithms, minutiae, i.e. endings and bifurcations of fingerprint ridges, are the most popular and widely used features for representation and matching of fingerprints [1].

There are two types of minutiae-based approaches: alignment-based global matching and local structure-based matching. The former uses explicit or implicit alignment to find the point correspondences [2, 3]. The latter attempts to match local structures which are constructed by the minutiae and related neighboring features $[4,5,6]$. In local matching approaches, each local structure is associated with structural properties invariant to translation and rotation, and the final matching score is calculated by accumulating the matching information of local neighborhood structures gradually. Therefore, local matching approaches usually have higher accuracy and efficiency. For example, the approach proposed by Chikkerur et al [6] uses star-like local structure called $K$-plet representation to encode local neighborhood of a minutia. Candidate corresponding star pairs are obtained through dynamic programming, and then a consolidation is followed based on a coupled breadth first search algorithm ( $C B F S$ ) to propagates the local matches simultaneously in both fingerprints to ensure a reliable matching of all minutiae.

Although the existing minutiae-based matchers exhibit good performance, the problem of fingerprint matching is still far beyond solved. It remains difficult to design a robust matching algorithm under some tough conditions, such as large nonlinear distortion, low quality of noisy fingerprint images, small overlap of partial fingerprints, etc. Much effort has been devoted to find solutions to cope with these difficulties, e.g. nonlinear deformation models [7], specialized enhancement methods [8], Level-3 features (e.g. pores) [9, 10].

In this paper, we propose a novel approach for fingerprint representation and matching, which is an improved version of the work by Chikkerur et al. [6]. In our approach, we defined a new feature Supplementary Associative Point (SAP) which is located at the associated ridge or valley of each minutia. By incorporating SAPs, our approach gives a more complete and compact graph-based representation, and also has a better performance with improved CBFS-based matching algorithm. Experimental results on FVC2002 databases are fair satisfied and showed that proposed approach outperforms the original algorithm.

The rest of this paper is organized as follows. Section 2 gives the detail description of proposed approach. Experimental results are reported in Section 3. Finally, a conclusion is drawn in Section 4. 


\section{Proposed Approach}

We present a novel algorithm for robust fingerprint recognition based on improved K-plet and CBFS which are proposed by S. Chikkerur et al at ICB'06 [6]. In this section, the improvements of our approach will be mainly described in detail, and we refer the reader to reference [6] for learning more about Chikkerur et al.'s work.

\subsection{Improved K-plet Based Representation}

Firstly, We define a new kind of feature called Supplementary Associative Point $(S A P)$ for fingerprint representation. As shown in Fig. 1, the SAP is extracted at a certain location at the associated ridge or valley of the corresponding minutia. The curve distance between the minutia and SAP, $d_{S A P}$ in Fig. 1, is set to the value of four times of average ridge distance empirically. Therefore, it greatly helps the minutia to represent the discriminative information in the neighborhood region around them. It should be pointed out that proposed SAP is different from Representative Ridge Point (RRP) proposed by Fang et al [11] in two aspects: (i) each minutia has only one determinative SAP, and (ii) the SAP corresponding to the minutia of ridge bifurcation is located at the associated valley.

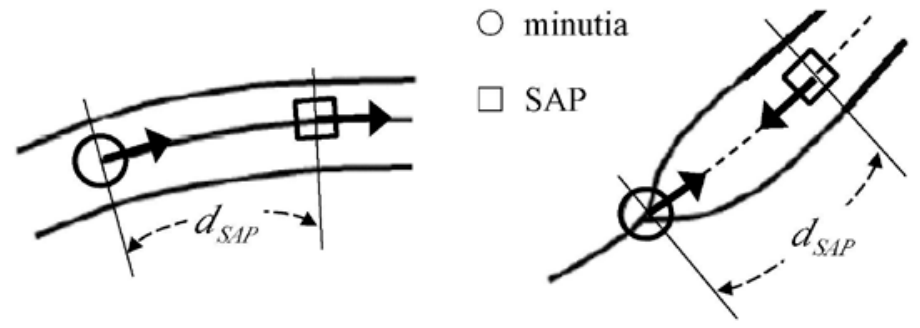

Fig. 1 Definition of supplementary associative point ( $S A P$ ) for minutiae with different types. The SAPs corresponding to the minutia with ridge ending (left) and bifurcation (right) are defined and extracted on the associate ridge and valley respectively

After the minutiae and SAPs are extracted, the improved K-plet (denoted by ImKplet) is constructed for each feature point (minutia or SAP). The ImKplet consists of a central point $p$ and $K$ other points $\left\{q_{1}, q_{2}, \cdots, q_{K}\right\}$ chosen from its local neighborhood. It should be noted that the point type (minutia or SAP) is not limited in proposed ImKplet. Each neighborhood point is defined in terms of its local radial coordinates $\left(\rho_{i}, \phi_{i}, \theta_{i}, \delta_{i}\right)$ as shown in Fig. 2. $\rho_{i}$ represents the 
Euclidean distance between $p$ and $q_{i}, \phi_{i}$ is is the relative orientation of point $q_{i}$ and the central point $p$. $\theta_{i}$ represents the direction of the edge connecting the two points. $\delta_{i}$ is true if $p$ and $q_{i}$ are the associated minutia and SAP, otherwise false.

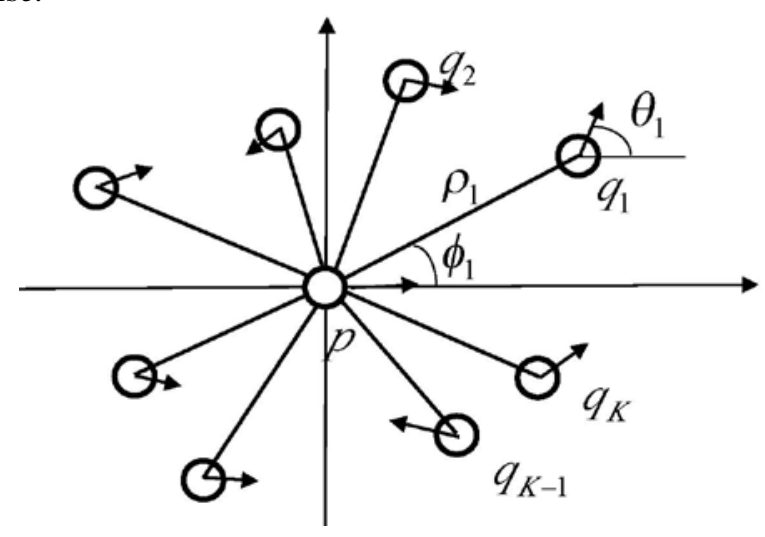

Fig. 2 The improved K-plet (ImKplet) and its local coordinate system. Note that $p$ and $q_{i}$ can be either minutia or SAP

The local structural relationship of the ImKplet can be encoded in the form of a graph $G(V, E)$ as similar as proposed in [6]. Each vertex $u$ represents a minutia or SAP and has attributes $\left(x_{u}, y_{u}, \theta_{u}, t_{u}\right)$. Each directed edge $(u, v)$ represents the neighboring relationship in ImKplet, and is labeled with the corresponding ImKplet coordinates $\left(\rho_{u v}, \phi_{u v}, \theta_{u v}, \delta_{u v}\right)$. Hence the graph $G$ provides an effective representation of the fingerprint. Figure 3 (a)-(f) demonstrate the graph-based representation of fingerprints based on K-plet and ImKplet.

\subsection{Improved CBFS Based Matching}

The matching algorithm in [6] firstly performs local neighborhood matching with a dynamic programming approach based on string alignment algorithm, then propagates the local matches to the K-plet of all the minutiae in the neighborhood successively through CBFS, and finally the match result with maximum number of vertex is considered to be the possible genuine correspondence. In proposed approach, the similar strategy for fingerprint matching is adopted. However, some improvements have been made according to the improved K-plet based representation. Here we emphasize the improvements as listed below, and refer the reader to reference [6] for details about CBFS-based matching algorithm. 


\subsubsection{Consistent matching between two corresponding minutia-SAP pairs}

As described in previous section, there are one-one correspondence between the minutiae and the related SAPs, and they have a close spatial connection. Therefore, the matching of two minutia-SAP pairs should be consistent and compatible. Based on this property, the following matching rules,

$$
\begin{gathered}
\text { NotMatched }(u, v) \Leftrightarrow \operatorname{NotMatched}\left(u_{S A P}, v_{S A P}\right) \\
\operatorname{Matched}(u, v) \Leftrightarrow \operatorname{Matched}\left(u_{S A P}, v_{S A P}\right)
\end{gathered}
$$

are hold for the two corresponding minutia-SAP pairs, $\left\langle u, u_{S A P}\right\rangle$ in query fingerprint and $\left\langle v, v_{S A P}\right\rangle$ in template fingerprint, when the nonlinear distortion in the local regions containing them is not extremely large.

\subsubsection{Local matching with fast pruning}

Although the size of feature point set is increased to twice as large as the original one, the trials of local neighborhood matching in proposed approach can be largely decreased fortunately. By utilizing the matching rule (Eq. 1), two ImKplets $\Gamma^{Q}$ and $\Gamma^{T}$ in the query and template fingerprints, centered with minutiae $u$ and $v$ respectively, would not be matched if the minutia-SAP pairs $\left\langle u, u_{S A P}\right\rangle$ and $\left\langle v, v_{S A P}\right\rangle$ are not matched. Therefore the further matching of $\Gamma^{Q}$ and $\Gamma^{T}$ are immediately avoided, and the computational burden of the matching algorithm is much reduced accordingly.

\subsubsection{Local match propagation scheme via SAP}

While consolidating all the local matches, Chikkerur et al's approach [6] uses coupled breadth-first search algorithm (CBFS) to successively propagate the local matches via the neighborhood minutiae in the K-plet. Besides the propagation procedure, our approach provide another novel and effective way to propagate the local matches by traveling along the graph edge of minutia-SAP pair according to the matching rule (Eq. 2). 


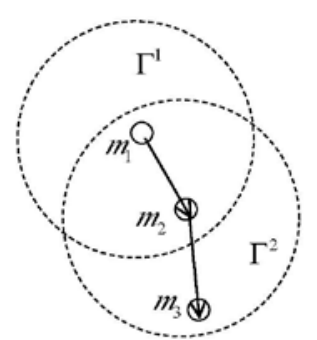

(a)

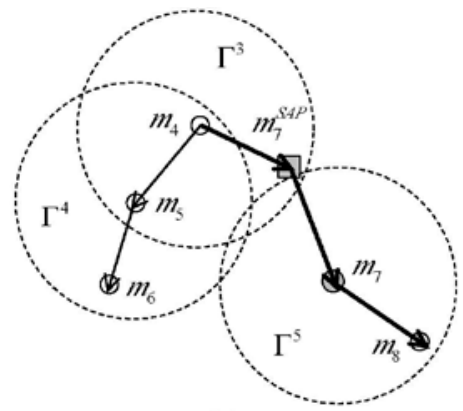

(b)

Fig. 3 Propagation schemes of local neighborhood match. (a) Chikkerur et al's approach [6], (b) Proposed approach

The above two propagation schemes are illustrated in Figure 3. As shown in Fig.3(a), Chikkerur et al's approach travels in the directed graph from K-plet $\Gamma^{1}$ to K-plet $\Gamma^{2}$ along the path $m_{1} \rightarrow m_{2} \rightarrow m_{3}$; thus it has a traversal distance $\left|\overrightarrow{m_{1} m_{2}}\right|+\left|\overrightarrow{m_{2} m_{3}}\right| \approx 2 r$, where $r$ is the average radius of the neighborhood of the K-plet. As shown in Fig.3(b), proposed approach can travel in the directed graph not only from ImKplet $\Gamma^{3}$ to ImKplet $\Gamma^{4}$ along the path $m_{4} \rightarrow m_{5} \rightarrow m_{6}$, but also from ImKplet $\Gamma^{3}$ to ImKplet $\Gamma^{4}$ along the path $m_{4} \rightarrow m_{7}^{S A P} \Rightarrow m_{7} \rightarrow m_{8}$, where the traversal along the graph edge of the minutia-SAP pair $m_{7}^{S A P} \Rightarrow m_{7}$ is the straight result of the matching rule (Eq. 2) and doesn't need to perform explicit local matching; thus it has a larger traversal distance $\left|\overrightarrow{m_{4} m_{7}^{S A P}}\right|+\left|\overrightarrow{m_{7}^{S A P} m_{7}}\right|+\left|\overrightarrow{m_{7} m_{8}}\right| \approx 2 r+d_{S A P}$. As we see from Fig. 3, by means of the minutia-SAP neighborhood of the ImKplet, the proposed matching algorithm could travel more far in the graph and obtain a wider matched subgraph.

\section{Experimental Evaluation}

To evaluate the performance, experiments are conducted on FVC2002 database [12]. Because the reference [6] did not present the detail of fingerprint image processing and minutiae extraction, a complete and direct comparison is not possible. For fair benchmark comparison, in our study, each dataset of FVC2002 is processed by a commercial AFIS software system VeriFinger 6.0 from Neurotechnologija [13]. After the fingerprint image is enhanced and thinned, the minutiae and SAPs are then extracted. 
Table 1 Performance comparison of EER on FVC2002 database

\begin{tabular}{cccc}
\hline Dataset & Original & Proposed & Improvement \\
\hline DB1 & $2.74 \%$ & $1.73 \%$ & $36.86 \%$ \\
DB2 & $2.19 \%$ & $1.72 \%$ & $21.46 \%$ \\
DB3 & $5.60 \%$ & $4.65 \%$ & $16.96 \%$ \\
DB4 & $4.11 \%$ & $3.23 \%$ & $21.41 \%$ \\
\hline Average & - & - & $24.17 \%$ \\
\hline
\end{tabular}

The overall comparative results of matching algorithms with respect to equal error rate (EER) are given in Table 1. The following formula,

$$
\text { improvement }=\frac{\text { original }- \text { proposed }}{\text { original }} \times 100 \%
$$

is used to calculate the improvement. From Table 1, it is observed that better performance is obtained by our approach on each dataset substantially, and an average improvement of $24.17 \%$ is achieved in total. In addition, the comparison of receiver operating characteristics (ROC) curves for each FVC2002 dataset are depicted in Fig. 4.
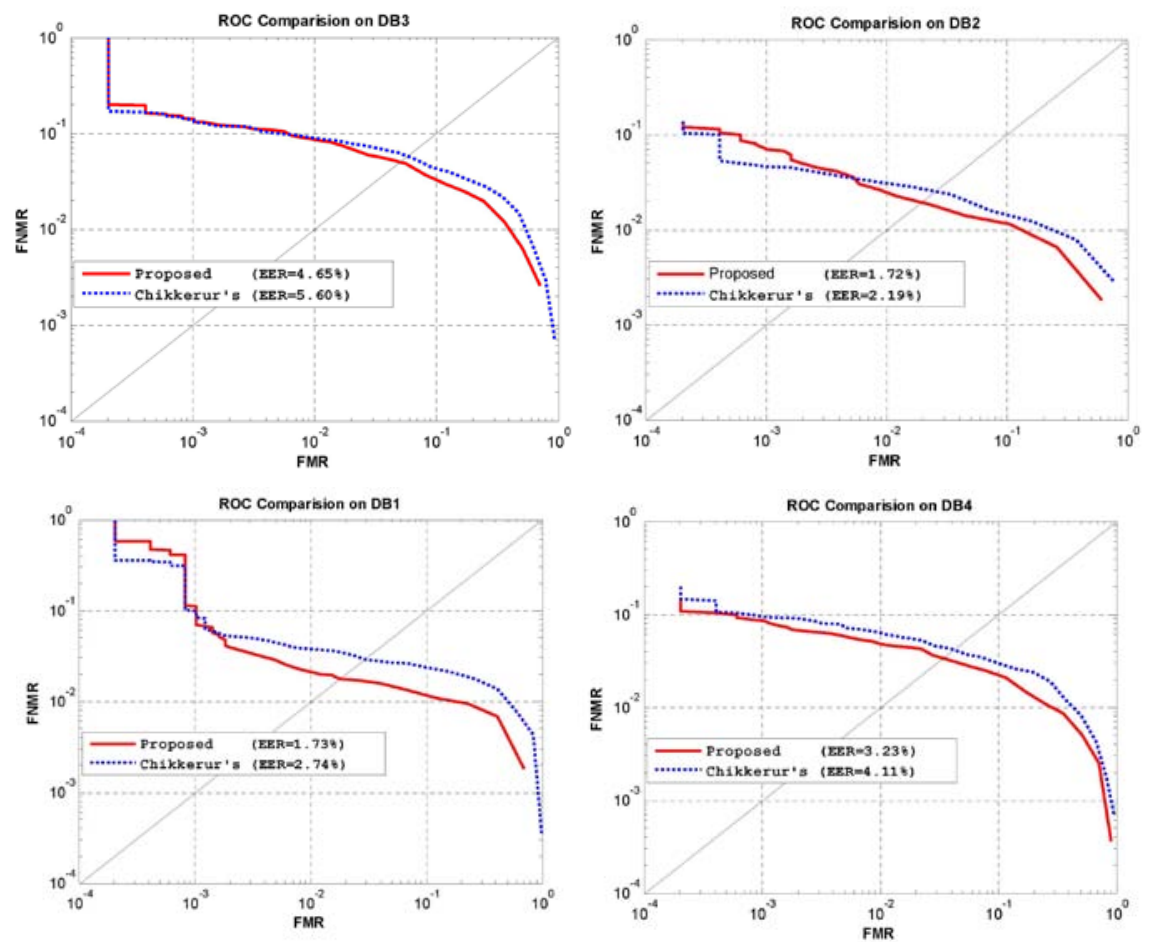

Fig. 4 A comparision of ROC curves for each dataset of FVC2002 
Figure 5 demonstrates the representation and matching of two sample fingerprints with large nonlinear distortion, “54_2” and “54_4”' in FVC2002 DB1. Figure 6 demonstrates the representation and matching of two sample fingerprints with partial overlap, "78_4" and "78_5" in FVC2002 DB1. As shown in these figures, our proposed representation covers a larger valid region and provides more discriminative information than Chikkerur et al's approach [6]. The compact and homogeneous form of the graph guarantees the excellent efficiency of the matching algorithm as the experimental results reveal.

In Figure 5, large nonlinear distortion can be observed in the left part (marked by dashed ellipses) of the fingerprint. For Chikkerur et al's approach [6], only about $30 \%$ of the minutiae can be correctly matched (see Fig. 5 (g)), and results in a low matching score 19.1. For our proposed approach, about $85 \%$ of the feature points including minutiae and SAPs are correctly matched, and results in a high matching score 43.6. We can see that proposed approach can easily cope with the nonlinear distortion existed in the whole fingerprint by traveling in the all directed graph of the fingerprint with the help of SAPs.

In Figure 6, the two genuine fingerprints have partial overlap in the upper part. By introducing more feature points such as SAPs, the partial fingerprints can perform a more reliable matching. From Fig. 6, we can see that proposed approach finds two more pairs of corresponding minutiae and a larger region correspondence than Chikkerur et al's approach.

\section{Conclusion}

We presented a novel algorithm for robust fingerprint recognition, which improves the approach "K-plet and coupled breadth-first search (CBFS)" proposed by Chikkerur et al at ICB'06.

For fingerprint representation, a new feature called supplementary associative point $(S A P)$ is defined, and local neighborhood structure improved K-plet (ImKplet) is constructed by the minutiae and SAPs. The local relationship of ImKplets is encoded in form of a directed graph which gives a complete and compact representation of the fingerprint.

For fingerprint matching, we proposed an improved CBFS-based matching algorithm. It utilizes the consistent matching property of the minutia-SAP pairs, and possesses advantages such as fast pruning of imposter local matches and deep propagation of local matches. By incorporating SAPs, the performance of proposed algorithm is much improved. Experiments on FVC2002 database showed that the proposed algorithm is strongly robust to nonlinear distortion and small partial overlap between the query and template fingerprints.

The experimental evaluation showed that the proposed algorithm outperforms Chikkerur et al's algorithm with an average improvement of $24.2 \%$ on equal error rate (EER). 


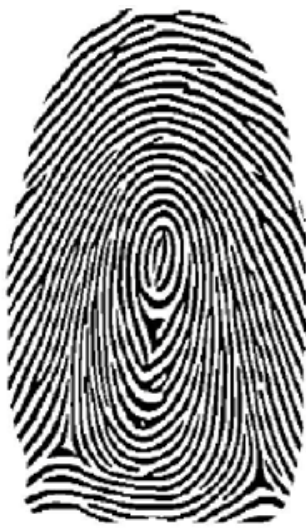

(a)

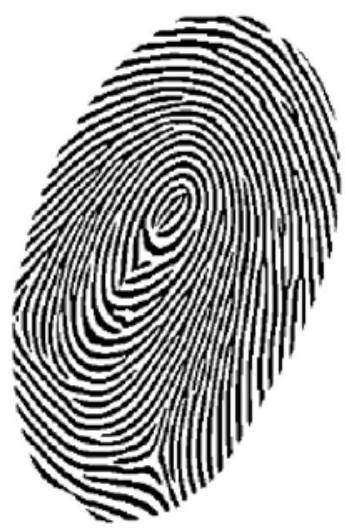

(d)

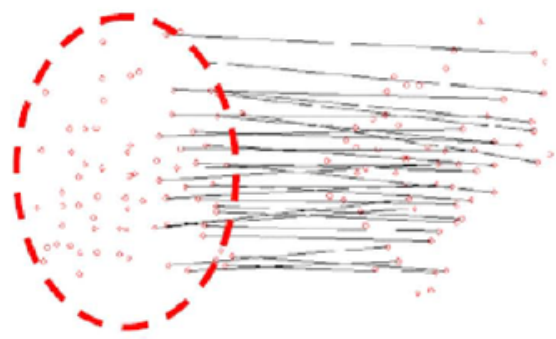

(g)

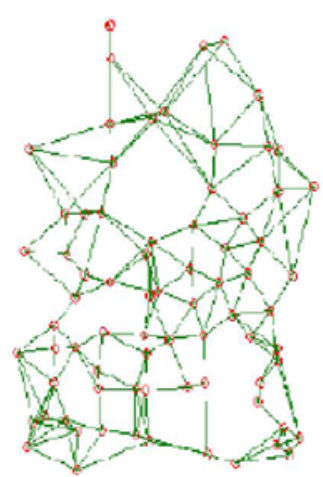

(b)

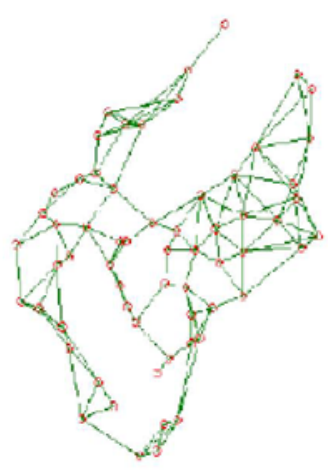

(e)

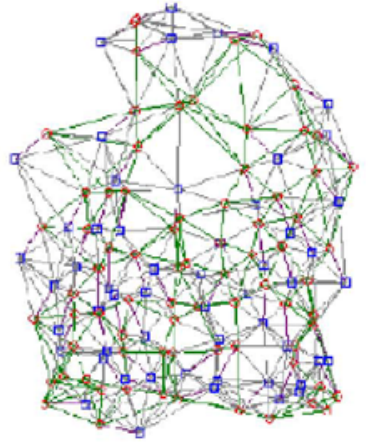

(c)

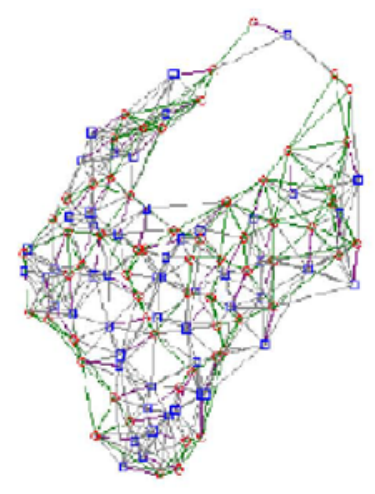

(f)

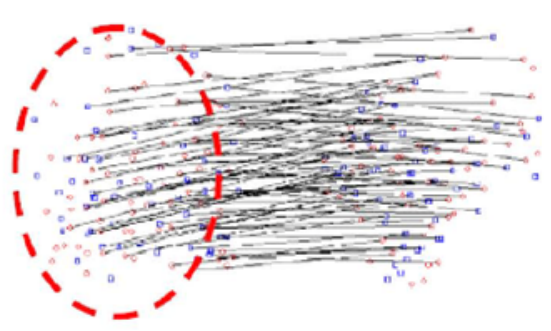

(h)

Fig. 5 An example of matching two fingerprints with large nonlinear distortion (circled out in (g) and (h)). (a) and (d): the two fingerprints "54_2" and "54_4" in FVC2002 DB1; (b) and (d): graphs constructed by K-plet [6]; (c) and (f): graphs constructed by proposed ImKplet; (g) and (h): match results of approach in [6] and proposed approach respectively 


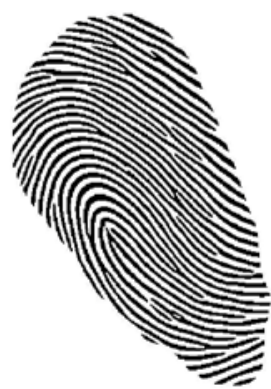

(a)

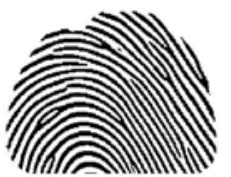

(d)

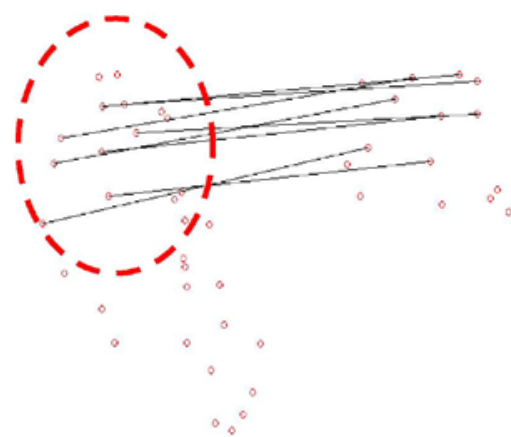

(g)

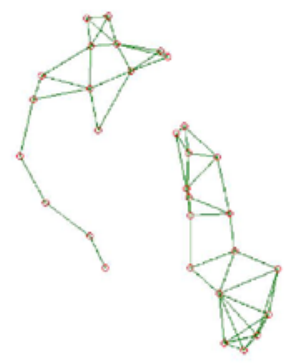

(b)

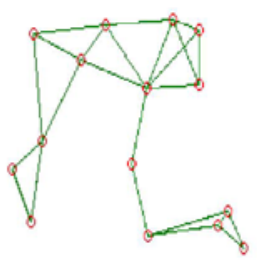

(e)

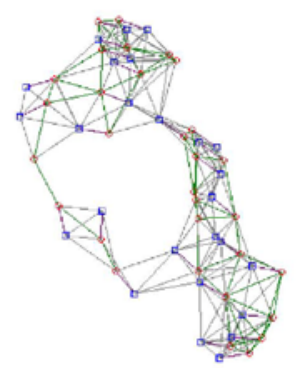

(c)

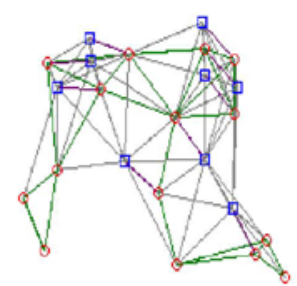

(f)

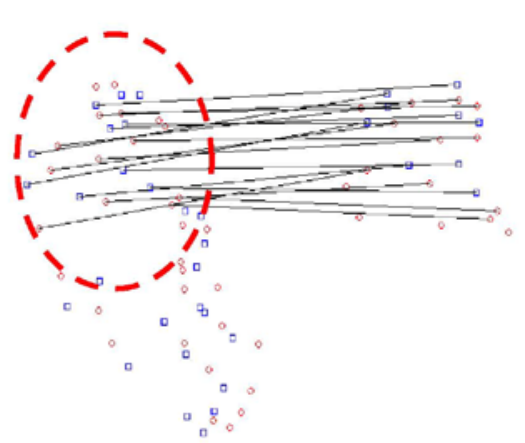

(h)

Fig. 6 An example of matching two fingerprints with partial overlap in the upper part (circled out in (g) and (h)). (a) and (d): the two fingerprints "79_4" and "78_5" in FVC2002 DB1; (b) and (d): graphs constructed by K-plet [6]; (c) and (f): graphs constructed by proposed ImKplet; (g) and (h): match results of approach in [6] and proposed approach respectively

Acknowledgments This work was supported by Beijing Outstanding Talents Project (No.2011D005002000003), Beijing Municipal Education Committee Surface Technology Development Plan (No.KM201210009012) and Scientific Research Start-Up Fund of Beijing Forestry University (Grant No. 2010BLX12). 


\section{References}

Maltoni, D., Maio, D., Jain, A. K., and Prabhakar, S. (2003) Handbook of Fingerprint Recognition. Springer, New York.

Jain, A. K., Hong, L., Pankanti, S., and Bolle, R. (1997) An identity authentication system using fingerprints. In Proc. IEEE, vol. 85 of 9, pp. 1365 - 1388.

Luo, X., Tian, J., and Wu, Y. (2000) A minutia matching algorithm in fingerprint verification. In Proc. of the 15th Int. Conf. on Pattern Recognition(ICPR'00), vol. 4, pp. 833 $-836$.

Jiang, X. D., and Yau, W. Y. (2000) Fingerprint minutiae matching based on the local and global structures. In Proc. of 15th Int. Conf. on Pattern Recognition (ICPR'00), pp. 1042 1045.

Jea, T.-Y., and Govindaraju, V. (2005) A minutia-based partial fingerprint recognition system. Pattern Recognition, 28:1672 - 1684.

S. Chikkerur, A. N. Cartwright, and V. Govindaraju. (2006) K-plet and coupled BFS: A graph based fingerprint representation and matching algorithm. In Proc. of Int. Conf. on Biometrics (ICB'06), pp.309 - 315.

Cappelli, R., Maio, D., and Maltoni, D. (2001) Modeling plastic distortion in fingerprint images. In Proc. of Int. Conf. on Advances in Pattern Recognition (ICAPR'01), pp. 369 376, Berlin Heidelberg, German. Springer-Verlag.

Ryu, C., Kong, S. G., and Kim, H. (2011) Enhancement of feature extraction for low-quality fingerprint images using stochastic resonance. Pattern Recognition Letters, 32(2):107 - 113. Jain, A. K., Chen, Y., and Demirkus, M. (2007) Pores and ridges: high-resolution fingerprint matching using level 3 features. IEEE Trans. on Pattern Analysis and Machine Intelligence, 29(1):15 - 27.

Zhao, Q., Zhang, D., Zhang, L., and Luo, N. (2010) High resolution partial fingerprint alignment using pore-valley descriptors. Pattern Recognition, 43:1050 - 1061.

Fang, G., Srihari, S. N., Srinivasan, H., and Phatak, P. (2007) Use of ridge points in partial fingerprint matching. In Biometric Technology for Human Identification IV: Proc. of SPIE, vol. 6539, pp. 65390D1 - 65390D9, Orlando, FL.

FVC2002: The 2nd Fingerprint Verification Competition. http://bias.csr.unibo.it/fvc2002/. 20, Sept., 2013.

VeriFinger SDK trials and demo versions, http://www.neurotechnology.com/download.html. 20, Sept., 2013. 